\title{
THE ASYMPTOTIC VALUE OF THE VOLUME OF A CERTAIN SET OF MATRICES
}

\author{
by HENRY JACK \\ (Received 28th January 1966)
}

\section{Introduction}

This paper is an appendix to a joint paper with Professor Macbeath. In (3), it was proved that the invariant measure, $m(k)$, of the set of real $n \times n$ matrices $\tau$, with determinant 1 and norm satisfying $\|\tau\| \leqq k$, had the property that

$$
\lim _{k \rightarrow \infty} \frac{m(\lambda k)}{m(k)}=\lambda^{n(n-1)}
$$

This last result was used by Macbeath and Rogers (5) to generalise from Riemann to Lebesgue integrable functions their version (4) of Siegel's mean value theorem.

The present paper shows that the invariant measure $l(k)$ of the same set of matrices, with the same norm, but with the additional condition that

has the property that

$$
\left\|\tau^{-1}\right\| \leqq k
$$

$$
\lim _{k \rightarrow \infty} \frac{l(\lambda k)}{l(k)}=\lambda^{\left[\frac{1}{2} n^{2}\right]},
$$

where $[x]$ denotes the integer part of the real number $x$. This means that the theorem of p. 322 of (5), modified to the new set of matrices, will go through practically word for word.

Added May 1966. Professor Macbeath tells me that the measure $l(k)$ introduced in this paper might be a candidate for the minimum in the product set problem for matrix groups; see (6).

\section{The reduction of $l(k)$ to a multiple integral}

The analysis of $\S \S 1-6$ of (3) is unaltered and the notations of $\S 7$ will be retained. The reader is reminded that as in that paragraph the case of $n+1$ variables is considered and that $\kappa=(n+1) \log k$.

The extra condition $\left\|\tau^{-1}\right\| \leqq k$, adds to line 9 of p. 219 of (3) the extra condition $x_{n} \geqq k^{-1}\left(x_{0} x_{1} \ldots x_{n}\right)^{1 / n+1}$. This becomes $y_{0}+y_{1}+\ldots+y_{n-1}-n y_{n} \leqq \kappa$ under the first change of variable of $\S 7$. Under the second change of variable of $\S 7$, this last inequality becomes $n z_{n}-z_{1}-z_{2}-\ldots-z_{n-1} \leqq \kappa$. 
After the integration with respect to $z_{0}$ is carried out, make the new change of variable $z_{1}=x_{1}, z_{r}-z_{r+1}=x_{r}(r=2,3, \ldots, n)$ and the problem is reduced to the evaluation of the multiple integral

$$
\phi_{n+1}(k)=\int_{C(\kappa)} \Delta\left(x_{1}, x_{2}, \ldots, x_{n}\right) d x_{1} d x_{2} \ldots d x_{n},
$$

where $\Delta\left(x_{1}, x_{2}, \ldots, x_{n}\right)$ denotes the $(n+1) \times(n+1)$ determinant whose typical row is $\left(1, \exp (n-2 r) x_{1}, \exp (n-2 r)\left(x_{1}+x_{2}\right), \ldots, \exp (n-2 r)\left(x_{1}+x_{2}+\ldots+x_{n}\right)\right)$, $r=0,1,2, \ldots, n$, and where $C(\kappa)$ is the region of all points $x=\left\{x_{1}, x_{2}, \ldots, x_{n}\right\}$ satisfying the inequalities

$$
\begin{gathered}
x_{1} \geqq 0, x_{2} \geqq 0, \ldots, x_{n} \geqq 0, \\
n x_{1}+(n-1) x_{2}+\ldots+(n+1-r) x_{r}+\ldots+x_{n} \leqq \kappa, \\
x_{1}+2 x_{2}+\ldots+r x_{r}+\ldots+n x_{n} \leqq \kappa .
\end{gathered}
$$

In $\S 8$ of (3) it was possible, since the corresponding $C(\kappa)$ was a simplex, to reduce the integral $\psi_{n+1}(k)$ to a contour integral, which by the theory of residues, is equal to a sum of terms of the type $\kappa^{r} e^{s \kappa}, r, s$ being integers.

In theory it is possible to express $C(\kappa)$ as a union of disjoint simplices. If this were done each integral could then be reduced to a contour integral by Lemma 2 of (2). However it appears to be difficult to obtain a systematic procedure which will find explicitly the coordinates of the vertices of such a union. Instead a unique vertex of $C(\kappa)$ will be distinguished, to be called the critical vertex. The simplex method from the theory of linear programming will then show that the value of $\kappa^{s} e^{r \kappa}$ at this critical vertex is the dominant term in $\phi_{n+1}(k)$; from this the proof of (1) is trivial.

\section{The vertices of $C(\kappa)$}

Let $\left(2^{\prime}\right),\left(3^{\prime}\right),\left(4^{\prime}\right)$ denote the equations derived from (2), (3), (4) when the inequality signs are replaced by equalities.

Each of the half spaces (2), (3), (4) are convex regions and since $C(\kappa)$ is their intersection, $C(\kappa)$ is also a convex region. The change of variable $x_{r} \rightarrow x_{n+1-r}$, $(r=1,2, \ldots, n)$, shows that $C(\kappa)$ is symmetrical about the hyperplane

$$
(n-1) x_{1}+(n-3) x_{2}+\ldots+(n+1-2 r) x_{r}+\ldots+(1-n) x_{n}=0
$$

passing through the origin and the intersection of $\left(3^{\prime}\right)$ and $\left(4^{\prime}\right)$.

The vertices of $C(\kappa)$ are among the solutions, $n$ at a time, of the $n+2$ equations $\left(2^{\prime}\right),\left(3^{\prime}\right),\left(4^{\prime}\right)$. One vertex determined by the equations $\left(2^{\prime}\right)$ is at the origin. Taking $\left(4^{\prime}\right)$ and any $n-1$ of $\left(2^{\prime}\right)$ gives as possible vertices, the points $x$, whose coordinate vectors are

$\{\kappa, 0,0, \ldots, 0\},\left\{0, \frac{\kappa}{2}, 0, \ldots, 0\right\}, \ldots,\left\{0, \ldots, 0, \frac{\kappa}{r}, 0, \ldots, 0\right\}, \ldots,\left\{0, \ldots, 0, \frac{\kappa}{n}\right\}$.

These vertices must of course also satisfy the inequality (3), so only those with $\frac{\kappa}{r}(n+1-r) \leqq \kappa$, or $n \geqq r \geqq \frac{1}{2}(n+1)$ are actual vertices. There is also the 
symmetrical set arising from ( $\left.3^{\prime}\right)$ and any $n-1$ of $\left(2^{\prime}\right)$. When $n$ is even, these two sets are distinct. When $n$ is odd, they have exactly one vertex in common, that in which the middle component, $r=\frac{1}{2}(n+1)$, is $\frac{2 \kappa}{n+1}$. This vertex lies in the hyperplane of symmetry of $C(\kappa)$; we shall call it the critical odd vertex.

All solutions of $\left(3^{\prime}\right)$ and $\left(4^{\prime}\right)$ and any $n-2$ of $\left(2^{\prime}\right)$ lie in the hyperplane of symmetry and will have all their components zero, except possibly two, say $x_{r}$ and $x_{s}$. These numbers satisfy $r x_{r}+s x_{s}=\kappa,(n+1-r) x_{r}+(n+1-s) x_{s}=\kappa$. When $r=s$, these equations have a solution if and only if $n$ is odd and $r=\frac{1}{2}(n+1)$. This gives the critical odd vertex. When $r \neq s$,

$$
x_{r}=\frac{\kappa(n+1-2 s)}{(n+1)(r-s)}, x_{s}=\frac{\kappa(2 r-n-1)}{(n+1)(r-s)} .
$$

These numbers must also satisfy all the inequalities (2), so only those solutions with $n \geqq r>\frac{1}{2}(n+1)>s \geqq 1$ are actual vertices. When $n$ is even, call the vertex with $r=\frac{1}{2} n+1, s=\frac{1}{2} n$ and so $x_{r}=x_{s}=\frac{\kappa}{n+1}$, the critical even vertex.

$C(\kappa)$ can be expressed as a union of simplices, disjoint apart from faces, as follows. The critical vertex can be "cut off" by a hyperplane which intersects $C(\kappa)$ in a flat polygon with $n$ vertices, such that these $n$ vertices and the critical vertex form a simplex $S_{N+1}$, which contains no other vertex of $C(\kappa)$. The remainder of $C(\kappa)$ is then " triangulated" into $N$ simplices $S_{r}, r=1,2, \ldots, N$.

\section{The reduction of $\phi_{n+1}(k)$ to a contour integral}

Expanding the determinant $\Delta\left(x_{1}, x_{2}, \ldots, x_{n}\right)$ by its first column gives

$$
\Delta=\sum_{\rho}( \pm) \exp \left\{\left(\alpha_{r}+\alpha_{s}+\ldots+\alpha_{t}+\alpha_{u}\right) x_{1}+\left(\alpha_{r}+\alpha_{s}+\ldots+\alpha_{t}\right) x_{2}+\ldots+\alpha_{r} x_{n}\right\},
$$

where $\rho=(r, s, \ldots, t, u)$ is a permutation of $(1,2, \ldots, n)$, the sign being positive for even permutations and negative for the odd. The entries in the row vector

$$
\alpha_{\rho}^{\prime}=\left(\alpha_{r}, \alpha_{r}^{\top}+\alpha_{s}, \ldots, \alpha_{r}+\alpha_{s}^{2}+\ldots+\alpha_{t}+\alpha_{u}\right)
$$

are the sums, the appropriate number of times, of the numbers $n, n-2, n-4$, $\ldots,-n$. Write $x$ for the column vector $\left\{x_{n}, x_{n-1}, \ldots, x_{1}\right\}$, then

$$
\Delta=\sum_{\rho}( \pm) \exp \left(\alpha_{\rho}^{\prime} x\right)
$$

Now let $v_{r, s}(r=1,2, \ldots, n+1 ; s=1,2, \ldots, N+1)$ be the column vector representing the $r$ th vertex of the sth simplex $S_{s}$ into which $C(\kappa)$ has been triangulated. As in $\S 2$ of (2) let $z_{r, s, \rho}=\alpha_{\rho}^{\prime} v_{r, s}$, then Lemma 2 of (2), shows that

$$
\int_{S_{s}} \exp \left(\alpha_{\rho}^{\prime} v_{r, s}\right) d x=\frac{C_{s} \kappa^{n}}{2 \pi i} \int e^{z} K_{\rho, s}(z) d z,
$$


round a suitable contour, where $C_{s}$ does not depend on $\kappa$, and

Thus

$$
\left(K_{\rho, s}(z)\right)^{-1}=\prod_{r=1}^{n+1}\left(z-z_{r, s, \rho}\right) \text {. }
$$

$$
\phi_{n+1}(k)=\sum_{s=1}^{N+1} \frac{C_{s} \kappa^{n}}{2 \pi i} \int e^{z} K_{\rho, s}(z) d z .
$$

It will be shown in the next paragraph that the maximum $M(k)$ of all possible $z_{r, s, \rho}$ is attained exactly once and at the critical vertex. By partial fractions and the residue theorem $\phi_{n+1}(k)$ is a sum of terms of the type $\kappa^{q} \exp \left(z_{r, s, \rho}\right)$. Thus as $k \rightarrow \infty$, the dominant terms is $\exp (M(k))$. From this, the proof of (1) is trivial.

\section{The maximum of $z_{r, s, p}$}

Since all points $x$ inside and on $C(\kappa)$ have non-negative coordinates, the maximum of all $\alpha_{\rho}^{\prime} x$, for fixed $x$ and varying $\rho$ must occur, when each entry in $\alpha_{\rho}^{\prime}$ is a maximum. Denote the maximum $\alpha_{\rho}^{\prime}$ by $\alpha^{\prime}$, then

$$
\alpha^{\prime}=(n, 2(n-1), \ldots, r(n+1-r), \ldots, 2(n-1), n) .
$$

By Theorem 3, p. 154 of (1), the maximum of $\alpha^{\prime} x$ for all $x$ inside and on $C(\kappa)$ is taken at a vertex. So in particular no point introduced for the triangulation of $C(\kappa)$ can give rise to the maximum.

For the vertices given by $\left(3^{\prime}\right)$ or $\left(4^{\prime}\right)$ and any $n-1$ of $\left(2^{\prime}\right)$ the value of $\alpha^{\prime} x$ is $r(n+1-r) \frac{\kappa}{r}=(n+1-r) \kappa$ and the maximum of this for $n \geqq r \geqq \frac{1}{2}(n+1)$ is $\frac{1}{2}(n+1) \kappa$ if $n$ is odd and $\frac{1}{2} n \kappa$ if $n$ is even. The maximum for $n$ odd is taken for $r=\frac{1}{2}(n+1)$ only, that is at the critical odd vertex.

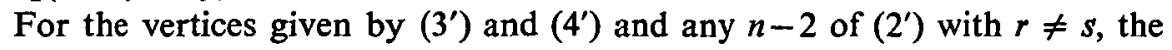
value of $\alpha^{\prime} x$ is

$$
r(n+1-r) x_{r}+s(n+1-s) x_{s}=\frac{2 \kappa}{n+1}\left\{\left(r-\frac{1}{2}(n+1)\right)\left(s-\frac{1}{2}(n+1)\right)+\frac{1}{4}(n+1)^{2}\right\} .
$$

Since $\left(r-\frac{1}{2}(n+1)\right)\left(s-\frac{1}{2}(n+1)\right)<0$ when $n$ is odd, the maximum of (5) is less than the maximum $\frac{1}{2}(n+1) \kappa$ found above for the other set of vertices. When $n$ is even, the maximum of (5) is taken when $r=\frac{1}{2} n+1, s=\frac{1}{2} n$ and has the value $\frac{n(n+2)}{2(n+1)} \kappa$, which is strictly greater than the maximum $\frac{1}{2} n \kappa$ for the other set of vertices. That is, for $n$ even, the maximum is taken at the critical even vertex.

Since $\kappa=(n+1) \log k$, it follows that

$$
M(k)=\left\{\begin{array}{l}
\frac{1}{2}(n+1)^{2} \log k \text { for } n \text { odd } \\
\frac{1}{2} n(n+2) \log k \text { for } n \text { even, }
\end{array}\right.
$$

and so that $\exp (M(k))=k^{\left[\frac{1}{2}(n+1)^{2}\right]}$. 


\section{REFERENCES}

(1) G. B. DANTzig, Linear Programming and Extensions (Princeton, 1963).

(2) H. JACK, An integral over the interior of a simplex, Proc. Edinburgh Math. Soc. (2) 13 (1962), 167-171.

(3) H. JACK and A. M. Macbeath, The volume of a certain set of matrices, Proc. Cambridge Phil. Soc. 55 (1959), 213-223.

(4) A. M. Macbeath and C. A. Rogers, A modified form of Siegel's mean value theorem, Proc. Cambridge Phil. Soc. 51 (1955), 565-576.

(5) A. M. Macbeath and C. A. Rogers, A modified form of Siegel's mean value theorem II, Proc. Cambridge Phil. Soc. 54 (1958), 322-326.

(6) A. M. MACвEATH, On the measure of product sets in a topological group, Jour. London Math. Soc. 35 (1960), 403-407.

\section{QueEN'S College}

DUNDEE 